[CONTRIbution from the Laboratory of THE George Washington University.]

\title{
A CONFIRMATION OF VAN'T HOFF'S HYPOTHESIS. OPTICAL SUPERPOSITION IN THE METASACCHARINS AND RELATED BODIES.
}

By FDWIN A. HILI

Received February 7, 1918

Van't Hoff's hypothesis, or the so-called principle of optical superposition, states that the specific rotation of an optically active substance is the algebraic sum of certain constants corresponding to the active atoms present.

The early work of Guye, Gautier and Walden, was supposed to afford experimental proof of the validity of this proposition, ${ }^{1}$ but Rosanoff ${ }^{2}$ and others have claimed to show, that owing to "serious errors in principle" involved in the experimental work, these results in reality prove nothing either for or against the theory, and more recently Patterson, ${ }^{3}$ in a series of 4 articles, has concluded "that the experiments of Guye and Walden have in reality no bearing on the matter in hand, and that the validity of Van't Hoff's hypothesis is distinctly open to doubt."

On the other hand the series of articles by Dr. C. S. Hudson ${ }^{4}$ which have appeared in ThIs JournaL from time to time, indicate that the principle does hold, at least approximately, in certain cases, for as he has remarked ${ }^{5}$ it is otherwise quite unlikely that the simple numerical relations disclosed by him could exist.

The problem may be more specifically stated as follows: The formula for $l$-arabinose is sometimes written as given below, with an aldehyde group at the end of the chain, indicating the presence of three active carbon atoms denoted by $\alpha, \beta$ and $\gamma$, respectively, so that the specific rotation of the molecule is the algebraic sum of the rotations of the active carbons $\alpha, \beta$ and $\gamma$.

$\mathrm{OH} \mathrm{OH} \mathrm{H}$

$$
\mathrm{HOCH}_{2}-\mathrm{C}-\mathrm{C}-\mathrm{C}-\mathrm{CH}-\mathrm{COH} \text {, }
$$

The disputed question is what will be the effect upon either the sign or the numerical value of the activity of any of these atoms, e.g., the $\alpha$ carbon if we allow the $\mathrm{H}$ and $\mathrm{OH}$ attached to any one of the other carbons, $e . g$., the $\beta$ or $\gamma$ carbon, to exchange places?

Van't Hoff's hypothesis predicates that there will be no change whatever, in either the sign or the magnitude of the activity of the $\alpha$ carbon; those like Rosanoff and Patterson, who question the validity of the hypo-

1 See Landolt, "Opt. Rot. of Org. Substances," pp. 296-298.

2 This JoUrnal, 28, 525 (I 906 ); 29, 536 (I907).

${ }^{3}$ J. Chem. Soc., 87, 33 (1905); 89, 1885 (1906); 91, 705 (1907); 107, 142 (1915).

- This JoURnal, 3I, 66-86 (1909); 32, 338-346 (1910); as also later articles.

'Ibid., 3I, 68 (1909), footnote. 
thesis, assert the possibility of a change in either the sign or numerical value or perhaps both of the activity of the $\alpha$ carbon under these circumstances.

In the above formula with three active carbons present, the theory requires the existence of 4 isomers, each having a different value of $[\alpha]_{D}$, and there are in fact 4 known pentose sugars, of which arabinose above-mentioned is one, the other 3 being, respectively, ribose, xylose and lyxose.

It would seem simple enough to let $\alpha, \beta$ and $\gamma$ represent the numerical values of the supposed constants, calling their signs + when $\mathrm{H}$ is above the chain and - when below it, and so to form 4 independent equations by using the value of $[\alpha]_{D}$ as determined for each sugar.

Then 3 of these equations could be used for determining the values of the constants, and if these values, when substituted in the fourth equation satisfied it within the limits of experimental error, the proposition would be fully established.

This method however fails when applied to the values of $[\alpha]_{D}$ for stable solutions of this group of sugars, for the fact that these sugars display mutarotation, is now generally held to indicate the presence of 4 , instead of 3 active atoms, and so to render the method inapplicable for lack of sufficient data.

And so with reference to all groups of isomers to which this method of proof can be applied, it will usually be found that we lack a sufficient number of determinations of $[\alpha]_{D}$ to compute the constants, and properly verify the values so computed, so that so far as the writer is advised, no case has yet been presented in which the values of all of the supposed constants can be computed, and then verified by the values of $[\alpha]_{D}$ not used in computing the constants, the values of $[\alpha]_{D}$ being for pure crystalline substances.

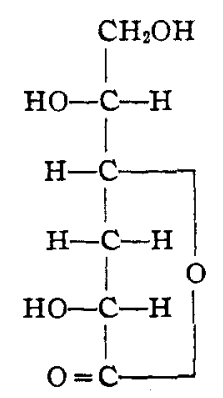

$\alpha, \delta$-Galacto-

metasaccharin.

(I).

\section{The Metasaccharins.}

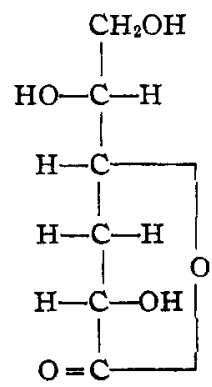

$\beta, \delta$-Galacto-

metasaccharin.

(II).

$-62.96^{\circ}$

55 to $60^{\circ}$<smiles>O=C1CO[C@H]([C@H](O)CO)[C@H]1C(O)CO</smiles>

$\beta$-Dextro-

metasaccharin.

(III).

$+8.2^{\circ}$

$92^{\circ}$

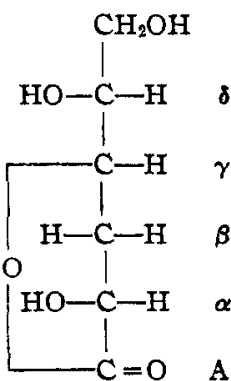

$\alpha$-Dextro-

metasaccharin.

(IV).

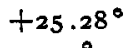

M. p. $=144^{\circ}$ 
The writer therefore presents herewith the cases of the 4 metasaccharins and their salts, in which the principle of optical superposition holds very closely, and to which he believes attention has never yet been called.

The formulas and data for these bodies will be found collected by Browne in his handbook of Sugar Analysis, p. 587 and 604. They were prepared by $\mathrm{Nef},{ }^{1}$ and the constants determined by him.

In these bodies only the $\alpha, \gamma$ and $\delta$ carbons are active, and the values of the 3 constants can therefore be computed from the values of $[\alpha]_{D}^{20}$ for the first 3 bodies, and from these constants the value of $[\alpha]_{D}^{20}$ for the fourth body can be computed, with a variation from the observed value less than the limit of experimental error, thus proving the principle for this set of isomers.

Thus let $\alpha, \gamma$ and $\delta$ be the constants of the $\alpha, \gamma$ and $\delta$ carbons, then by Van't Hoff's principle we shall have for any one of these bodies

$$
[\alpha]_{D}^{20}= \pm \alpha \pm \gamma \neq \delta
$$

For any particular body I shall, for vertically written formulas, give the constants the + sign when the $\mathrm{H}$ atom is on the right hand, and the - sign when on the left hand of the vertical chain, the A carbon being at the bottom we then have available these equations.

From $\alpha, \delta$-galacto metasaccharin $[\alpha]_{D}^{20}=+\alpha-\gamma+\delta=45.3^{\circ 2}$
From $\beta, \delta$-galacto metasaccharin $[\alpha]_{D}^{20}=-\alpha-\gamma+\delta=-62.96^{2}$
From $\beta$-dextro metasaccharin $[\alpha]_{D}^{20}=-\alpha+\gamma+\delta=+8.2^{3}$
From $\alpha$-dextro metasaccharin $\quad[\alpha]_{D}^{20}=+\alpha+\gamma+\delta=+25.28^{3}$

We can select any 3 of these equations, and having determined the constants therefrom, insert their values in the fourth equation, and thereby compute the value of $[\alpha]_{D}^{20}$. Thus using the first three equations, the values of the constants are as follows:

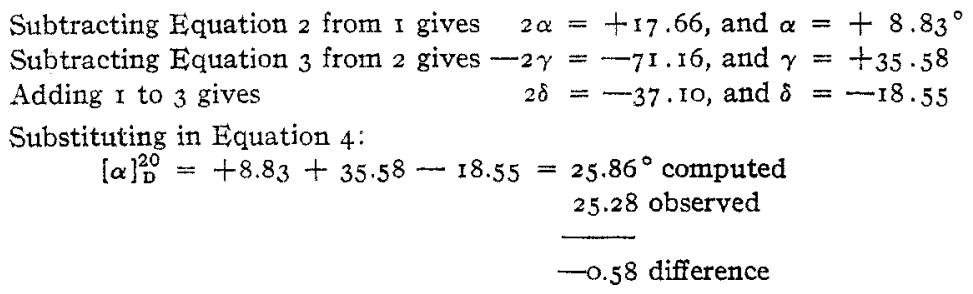

This fully establishes the principle.

\section{Recomputation of the Constants.}

By using all 4 of the observed values of $[\alpha]_{D}^{20}$, and giving each observation an equal weight, so as to distribute the errors of observation more equally between the values of the constants, we obtain the following values for the

\footnotetext{
${ }^{1}$ Ann., 376, x (r910).

2 Browne, p. 604 .

3 Browne, p. 587 ; citing Nef, $A n n ., 376$, I (1910).
} 
three constants, specific rotations being stated to the nearest tenth of a degree.

Constants OF THE METASACChaRINS.

\begin{tabular}{|c|c|c|c|}
\hline \multirow{2}{*}{ Rotations. } & \multicolumn{3}{|c|}{ Values of the constant. } \\
\hline & $\alpha$. & $\gamma$ & $\delta$. \\
\hline & $\begin{array}{l}+8.9^{\circ} \\
+1426^{\circ}\end{array}$ & $\begin{array}{l}+35.5^{\circ} \\
+5767^{\circ}\end{array}$ & $\begin{array}{l}-18.8^{\circ} \\
-3046^{\circ}\end{array}$ \\
\hline
\end{tabular}

Using these values, we can compute the following table of specific rotations:

Computed SPECIFIC Rotations of the MEtasaccharins.

Bodies. $\quad$ Signs of constants. Equations. $\overbrace{\text { Computed. Observed. Difference. }}^{\text {Values of }[\alpha]_{\mathrm{D}}^{20} \text {. }}$

$\alpha, \delta$-Galactometasaccharin $+\alpha-\gamma+\delta+8.9-35.5-18.8^{\circ}-45.4^{\circ}-45.3^{\circ} \rightarrow 0$. ro ${ }^{\circ}$

$\beta, \delta$-Galactometasaccharin $-\alpha-\gamma+\delta-8.9-35.5-18.8-63.2-62.96-0.24$

$\beta$-Dextrometasaccharin $-\alpha+\gamma+\delta-8.9+35.5-18.8+8 . \alpha+8.20-0.20$

$\alpha$-Dextrometasaccharin $+\alpha+\gamma+\delta+8.9+35.5-18.8+25.6+25.28+0.32$

The agreement between computed and observed values, as shown by the differences in the last column, being well within the limit of experimental error.

The metasaccharins are in structure lactones of acids, and when they form salts, with organic bases they act as such, and must hence contain a carboxyl group, which is evidently formed by the opening of the ring, and the addition of a molecule of water, the hydroxyl of which attaches to the $\gamma$ carbon, and the hydrogen to the oxygen of the broken ring, forming the carboxyl group.

The alkaloids strychnine, brucine and quinine are nitrogen bases, forming salts by addition instead of subtraction, the first two being monacid, and the latter diacid.

To what extent the lactonic ring is broken up and the corresponding acid formed, when a metasaccharin is dissolved in water, I am not advised. Neff, ${ }^{1}$ who prepared these metasaccharins by the action of alkalies on different sugars, also prepared their strychnine, brucine and quinine salts and determined the values of $[\alpha]_{D}^{20}$ in aqueous solution for all of them. ${ }^{2}$ By using these values it is found, that for each of these three bases, the 4 corresponding salts of the metasaccharins form a similar series, in which the principle of optical superposition also holds, as is shown below.

\section{The Strychnine Salts of the Metasaccharin.}

The formula for these bodies may be written

$$
[\alpha]_{\mathrm{D}}= \pm \alpha \pm \gamma \pm \delta \pm \text { St. }
$$

Where St is the sum of the effects of the active atoms of the strychnine nucleus, giving us the following four equations:

${ }^{1}$ Ann., 376, I (1910).

${ }^{2}$ Loc, cit. 


\section{$[\alpha]_{D}^{20}$ FOR STRYChNINE SALTS}

$\alpha, \delta$-Galactometasaccharin $=-8.41^{\circ}=+\alpha-\gamma+\delta \pm \mathrm{St}^{1}$

$\beta, \delta$-Galactometasaccharin $=-23.43=-\alpha-\gamma+\delta=\mathrm{St}^{1}$

$\alpha$-Dextrometasaccharin $=-19.50=+\alpha+\gamma+\delta \pm \mathrm{St}^{1}$

$\beta$-Dextrometasaccharin $\quad=-30.79=-\alpha+\gamma+\delta \pm \mathrm{St}^{1}$

Subtracting 6 from 5 gives $2 \alpha=+15.02^{\circ}$ and $\alpha=+7.51^{\circ}$

Subtracting 7 from 5 gives $-2 \gamma=+_{11} .09$ and $\gamma=-5.55$

Adding 6 to 7 gives $\quad 2 \delta \pm 2 S t=-42.93$ and $\delta \neq S t=-21.47$

Substituting these values in Equation 8 :

$$
\begin{aligned}
-\alpha+\gamma+\delta \pm S t=-7.51-5.55-21.47= & -34.53 \text { computed } \\
& -30.79 \text { observed } \\
& -3.74 \text { difference }
\end{aligned}
$$

This is a difference which is within the limits of experimental error.

This difference, as before, can be distributed between the values of the 3 constants, by reducing each constant numerically by the quantity $0.93^{\circ}$, giving for the values of the constants for the strychnine salts as follows:

Constants for Strychnine Salts.

Rotations.

$$
\begin{array}{ccc}
\alpha . & \gamma & \delta \neq \text { st. } \\
+6.58^{\circ} & -4.62^{\circ} & -20.54^{\circ} \\
+3382 & -2375 & -1055^{\circ}
\end{array}
$$

Specific rotation $[\alpha]_{\mathbf{D}}^{20}$

Molecular rotation $[\mathrm{M}]_{\mathbf{p}}^{20}$.

Using the above values of the constants for the specific rotation, we have the following table:

Comptted Specific Rotations of Strychnine Salts of the Metasaccharins.

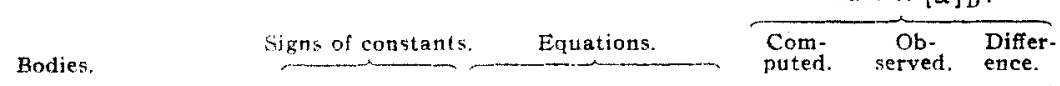

$\alpha, \delta$-Galactometasaccharin $+\alpha-\gamma+\delta \pm S t+6.58+4.62-20.54-9.34^{\circ}-8.41^{\circ}-0.93^{\circ}$

$\beta, \delta$-Galactometasaccharin $-\alpha-\gamma+\delta \pm S t-6.58+4.62-20.54-22.50-23.43+0.93$

$\alpha$-Dextrometasaccharin $+\alpha+\gamma+\delta=S t+6.58-4.62-20.54-18.58-19.50 \quad+0.92$

$\beta$-Dextrometasaccharin $-\alpha+\gamma+\delta \pm 5 t-6.58-4.62-20.54-31.74-30.79 \longrightarrow 0.95$

Here again the agreement between the observed and computed values of $[\alpha]_{\mathrm{D}}$ is good, and the principle is fully established.

\section{The Brucine Salts of the Metasaccharins.}

The formula for these bodies will be

$$
[\alpha]_{D}= \pm \alpha \pm \gamma \neq \delta \neq \mathrm{Br} \text {. }
$$

Where as before $\mathrm{Br}$ is the sum of the constants for the active atoms of the brucine molecule, the 4 equations being:

$$
[\alpha]_{D}^{20} \text { FOR BRUCINE SALTS. }
$$

$$
\begin{aligned}
& \alpha, \delta \text {-Galactometasaccharin }=+\alpha-\gamma+\delta=\mathrm{Br}=-12.74^{\circ} \\
& \beta, \delta \text {-Galactometasaccharin }=-\alpha-\gamma+\delta \neq \mathrm{Br}=-25.20 \\
& \alpha \text {-Dextrometasaccharin }=+\alpha+\gamma+\delta \pm \mathrm{Br}=-23.14 \\
& \beta \text {-Dextrometasaccharin }=-\alpha+\gamma+\delta=\mathrm{Br}=-33.14
\end{aligned}
$$

${ }^{1}$ Nef, Ann., 376, 74, 81, 98 and 97 (19ro). 
The values of $[\alpha]_{D}^{20}$ are those given by Nef. ${ }^{1}$ Following the method used for the strychnine salts, we can obtain from Equations 9, ro and II the values $\alpha=+6.24^{\circ}, \gamma=-5.2^{\circ}$ and $(\delta \pm \mathrm{Br})=-24.18^{\circ}$, whence by substitution in Equation i2 we have

$$
\begin{aligned}
-\alpha+\gamma+(\delta \neq \mathrm{Br})=-6.24-5.2-24.18= & -36.62^{\circ} \text { computed } \\
& -34.14 \text { observed } \\
& -2.42 \text { difference }
\end{aligned}
$$

This is a difference which is not in excess of the experimental error, and which when distributed as before equally between the 4 observations, reduces the average difference to $0.62^{\circ}$, and gives us the following constants for the brucine salts:

CONSTANTS FOR BRUCINE SALTS.

Rotations.

Specific rotations $[\alpha]_{D}^{20}$

Molecular rotations $[\mathbf{M}]_{\mathbf{D}}^{20}$ $\alpha . \quad \gamma . \quad \delta \pm \mathrm{Br}$.

Then as before, using the corrected values of the constants for the specific

\begin{tabular}{|c|c|c|c|c|c|}
\hline \multirow[b]{2}{*}{ Bodies. } & \multirow[b]{2}{*}{ Signs of constants. } & \multirow[b]{2}{*}{ Equations. } & \multicolumn{3}{|c|}{ Values of $[\boldsymbol{\alpha}]_{\mathrm{D}}^{20}$. } \\
\hline & & & $\begin{array}{l}\text { Com- } \\
\text { puted. }\end{array}$ & $\begin{array}{c}\text { Ob- } \\
\text { served. }\end{array}$ & $\begin{array}{l}\text { Differ- } \\
\text { ence. }\end{array}$ \\
\hline $\begin{array}{r}\text { z, } \delta \text { - Galactometa- } \\
\text { saccharin....... }\end{array}$ & $+\alpha-\gamma+(\delta \pm \mathrm{Br})$ & $+5.62+4.58-23.56$ & $-13.36^{\circ}$ & $-12.74^{\circ}$ & $-0.62^{\circ}$ \\
\hline $\begin{array}{r}3, \delta \text { - Galactometa- } \\
\text { saccharin....... } \\
x \text { - Dextrometa- }\end{array}$ & $-\alpha-\gamma+(\delta \pm \mathrm{Br})$ & $-5.62+4.58-23.56$ & -24.60 & -25.20 & +0.60 \\
\hline $\begin{array}{l}\text { saccharin....... } \\
\text { - Dextrometa- }\end{array}$ & $+\alpha+\gamma+(\delta=\mathrm{Br})$ & $+5.62-4.58-23.56$ & -22.52 & -23.14 & +0.62 \\
\hline
\end{tabular}
rotation, we obtain the following table:

Computed Specific Rotations, Brucine Salts of Metasaccharins

Here is an agreement between observed and computed values which is even better than in the case of the strychnine salts.

IV. The Quinine Salts of the Metasaccharins.

The formula for these bodies will be

$$
[\alpha]_{D}= \pm \alpha \pm \gamma \neq \delta \pm Q u
$$

where as before $Q u$ is the sum of the constants for the active atoms of the quinine molecule, the 4 equations being

$[\alpha]_{D}^{20}$ FOR QUININE SALTS.

$$
\begin{array}{ll}
\alpha, \delta \text {-Galactometasaccharin } & =+\alpha-\gamma+\delta \neq Q \mathrm{u}=-90.5^{\circ} \\
\beta, \delta \text {-Galactometasaccharin } & =-\alpha-\gamma+\delta \neq Q \mathrm{u}=-\mathrm{I04.I} \\
\alpha \text {-Dextrometasaccharin } & =+\alpha+\gamma+\delta \neq Q \mathrm{u}=-\mathrm{I00.9} \\
\beta \text {-Dextrometasaccharin } & =-\alpha+\gamma+\delta \neq \mathrm{Qu}=-\mathrm{II3.6}
\end{array}
$$

1 Loc. cit. 
The values of $[\alpha]_{D}^{20}$ are those given by Nef. ${ }^{1}$

Then as before, from Equations $\mathrm{I}_{3}, \mathrm{I}_{4}$ and $\mathrm{I}_{5}$ we can obtain the values $\alpha=+6.8, \gamma=-5.2$ and $\delta \pm Q u=-90.5$.

Whence by substitution in Equation 16 we have

$$
\begin{aligned}
-\alpha+\gamma+(\delta \pm Q u)=-6.8-5.2-\mathrm{IO} 2.5= & -\mathrm{Ir} 4.5 \text { computed } \\
& -\mathrm{II} 3.6 \text { observed } \\
& -0.9 \text { difference }
\end{aligned}
$$

This is a very satisfactory proof of the application of the principle.

When this difference of $-0.9^{\circ}$ is distributed between the 4 observations, we obtain the following values for the constants:

CONSTANTS FOR QUININE SALTS.

Rotation.

Specific rotation $[\alpha]_{0}^{20}$

Molecular rotation $[\mathrm{M}]_{\mathrm{D}}^{20}$

\section{$\alpha$.}

$\gamma . \quad \delta \pm Q u$.

$+6.58^{\circ}-4.98^{\circ}-102.28^{\circ}$

$+3316-2510-51549$

From which constants the following table is computed:

Computed Specific Rotations, Quinine Salts of Metasaccharins.

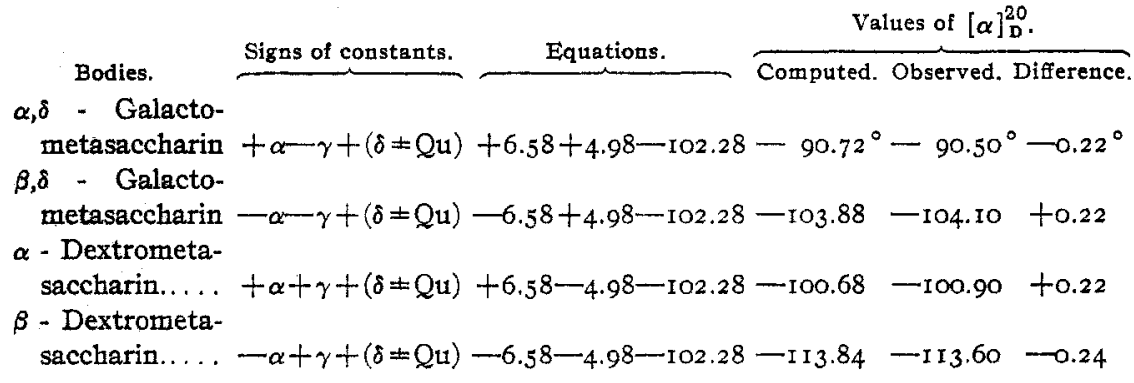

This shows etceedingly good agreement between computed and observed values.

Below will be found a comparative table of the constants of these metasaccharins and their salts.

Computed Constants of the Metasacchartins and Thetr Salts Specific and MOLECULAR ROtations.

Bodies. $\quad \overbrace{\text { Base. Total. }}^{\begin{array}{c}\text { Molecular } \\ \text { weights. }\end{array}} \overbrace{\alpha,}^{\text {Specific rotations }[\alpha]_{D} .} \overbrace{\gamma .}^{\text {Moleculat rotations }[M]_{D}}$.

Metasaccharins...... $180+8.8^{\circ}+35.6^{\circ}-18.8^{\circ}+1426^{\circ}+5767^{\circ}-3046^{\circ}$

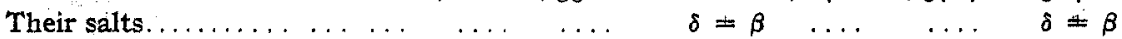

Strychnine...... $334514+6.58-4.62-20.54+3382-2375-10588$

Brucine......... $394574+5.62-4.58-23.56+3226-2629-13523$

Quinine acid salt... $324504+6.58-4.98-102.28+3316-25$ 10 -51549

As the molecular weights of the substances differ, because of the difference in the molecular weights of the 3 bases, we would not expect the values of the $\alpha$ and $\gamma$ constants of specific rotation to agree. The molecular

'Loc. cit. 
rotation constants, however, should agree for the $\alpha$ and $\gamma$ carbons, provided the various salts are all dissociated to an equal extent, for then the effect of the base would be fully eliminated by subtraction.

It will be noted, that the agreement between the three salts in the values of the molecular constants of the $\alpha$ and $\gamma$ carbons, is quite good, the average for the $\alpha$ constant being $+3308^{\circ}$, and for the $\gamma$ constant $-2805^{\circ}$. The departures from the average values being for the $\alpha$ constants, $+74^{\circ},+8^{\circ}$ and -82 , and for the $\gamma$ constants, $+135^{\circ},-5^{\circ}$ and $-119^{\circ}$, which are less than $1 / 2$ degree of specific rotation in the $\alpha$ constant, and less than a single degree of such rotation in the $\gamma$ constant, and may be considered well within the limit of experimental error.

The metasaccharins themselves are ring compounds while the salts are chains, and we therefore find as would be expected considerable difference in the values of the constants for the acids on the one hand, and for the salts on the other. The value of the $\delta$ constant in the case of the salts above, is tied up with the activity of the active base, but we have already found the following values, viz.:

$$
\delta \pm \mathrm{St}=-\mathrm{ro558}, \delta \pm \mathrm{Br}=-\mathrm{r} 3523, \delta \pm \mathrm{Qu}=-5 \mathrm{I} 549
$$

Now by using these molecular constants, with the constants for the $\alpha$ and $\gamma$ carbons, and using the equations already established, the molecular and specific rotations of all of the 12 salts can be computed with very small departures from the observed values, as is shown in the following table:

Salts of the Metasaccharins. Computations of Molecular and Specific ROTATIONS.

Molecular constants. Specific rotations.

Bodies Equation. $\overbrace{\alpha .} \gamma_{\delta} \delta \beta$. Motation. Computed. Observed. Diff. Strychnine Salts of the Metasaccharins $M . W=514$.

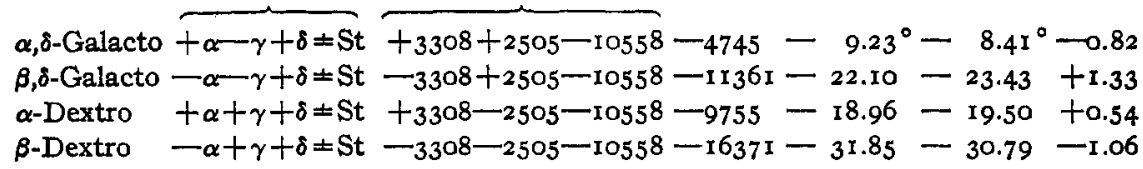

Brucine Salts of the Metasaccharins M. W $=574$.

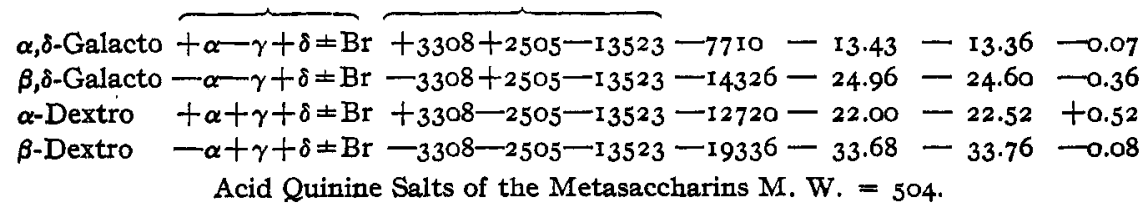

$\alpha, \delta$-Galacto $\overbrace{+\alpha-\gamma+\delta=Q u} u \overbrace{+3308+2505-51549}-45736-90.74-90.50-0.24$ $\beta, \delta$-Galacto $-\alpha-\gamma+\delta=Q u-3308+2505-51549-52352-103.87-104.10$ to.23 $\alpha$-Dextro $+\alpha+\gamma+\delta=Q u+3308-2505-51549-50746-100.68-100.90 \quad+0.22$ $\beta$-Dextro $-\alpha+\gamma+\delta \pm Q u-3308-2505-51549-57362-113.81-{ }_{113.60}-0.21$

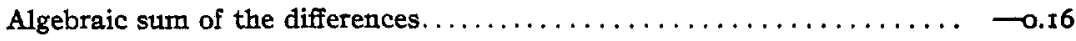
Average departure $\ldots \ldots \ldots \ldots \ldots \ldots \ldots \ldots \ldots \ldots \ldots \ldots \ldots \ldots \ldots .47$ 


\section{The Work of Dr. C. S. Hudson.}

In view of the closeness of the application of Van't Hoff's principle to the metasaccharins and their salts, as has been already illustrated, I will briefly review the work of Dr. C. S. Hudson and his co-workers, which apparently indicates the application of the principle to various other groups of substances as well.

In I909 Dr. Hudson ${ }^{1}$ announced that the specific rotation of many sugars and their derivatives could be expressed by the equation $[\alpha]_{D}=B \pm A$ where $A$ represented the rotation of the end asymmetric carbon, and $B$ the algebraic sum of the rotations of the remaining active carbons present. In this and subsequent papers he proved that $A$ remained constant, notwithstanding large changes in the value of $B$ due to changes of configuration, of these remaining active carbons. This would certainly indicate that in all of these cases, some 30 or more, the activity of the A carbon was changed neither in value nor sign, by configuration changes in the balance of the molecule. ${ }^{2}$

In most of these cases the agreement was well within the limits of experimental error.

These investigations clearly indicate the establishment of Hudson's original proposition, that in the case of a pair of multirotating sugars, reaching an equilibrium in solution, the rotations, either molecular or specific, are the algebraic sum of two constants $A$ and $B$ so that $[\alpha]_{D}=$ $\mathrm{B} \pm \mathrm{A}$ where $\mathrm{A}$ is a constant corresponding to the end asymmetric carbon, its sign depending upon the configuration of the $A$ carbon and $B$ is the joint effect of all of the remaining asymmetric atoms in the molecule.

Changes in the value of $\mathrm{B}$, caused by changes of configuration of the $\alpha$, $\beta, \gamma, \delta$, etc., carbons, do not change the constant difference of ${ }^{\top} \mathrm{A}$, and so do not affect the activity of the $A$ carbon in either magnitude or sign. This principle of Hudson's has been universally accepted, and is held by such workers as Dr. Levene of the Rockefeller Institute of Medical Research ${ }^{3}$ to afford "telling evidence in support of the principle of optical superposition." It should be further noted that Hudson's work establishes the principle for other carbons than the end carbon A. Thus for instance the 2

1 This Journal, 31, 69 (1909).

2 For the use of those interested the following references to some of this work are given below: Tris JournaL, 3I, 69 (r9o9), glucose, galactose, arabinose and lactose; Ibid., 36, 1265 (1914), glucosepentacetate and tetracetyl, methylglucoside; Ibid., 37, 1275 (1915), xylosetetracetate, glucosepentacetate and lactose octacetate; Ibid., 37, 1276 (I9I5), maltose, cellose and lactose octacetates; Ibid., 38, I434 (1916), glucosamine and chondrosamine pentacetates; $I$ bid., $3^{8}$, I576 (I 9 16), hexacetylglucoheptose, glucosepentacetate, and maltose pentacetate; Ibid., 39, I035 (I917), thirteen miscellaneous aldose sugars; J. Ind. Eng. Chem., 8, 379 (1906), monomethylglucose and glycol glucosides.

$$
\text { 3. Biol. Chem., 23, 145 (I915). }
$$


sugars $\alpha, \delta$-glucose and $\alpha, \delta$-galactose differ only in the configuration of the $\gamma$ carbon, the difference of their specificrotations being $+\mathrm{Ir} 3.4^{\circ}-\mathrm{I} 44^{\circ}=-30.6$ as the value of $2 \gamma$. If now we change the configuration of the $A$ carbon of these 2 sugars by reversing the positions of $\mathrm{H}$ and $\mathrm{OH}$ we have the $\beta$ forms of both sugars thereby produced, but the difference between $\beta, \delta$ glucoseand $\beta, \delta$-galactose remains practically unchanged being $+19^{\circ}-52^{\circ}=$ $-33^{\circ}$ for $2 \gamma$ against $-30.6^{\circ}$ for $2 \gamma$ as previously obtained, showing that changing the configuration of the $\mathrm{A}$ carbon has no affect upon either the magnitude or sign of the constant of the $\gamma$ carbon.

\section{Summary.}

It has been shown, not only for the 4 metasaccharins but also for their strychnine, brucine and quinine salts:

First, that their specific and molecular rotations are in each case the algebraic sums of three constants corresponding to the 3 active atoms present, the signs of the constants being taken + or - with $\mathrm{H}$ to the right or left in vertically written formulas with the A carbon below thus proving the validity of the principle of optical superposition in these cases.

Second, that the constants are practically identical for the $\alpha$ carbon in the three classes of salts, and likewise for the $\gamma$ carbon, but differ considerably from the $\alpha$ and $\gamma$ constants for the saccharins themselves, which was to have been expected in view of the fact that ring structure is present in the saccharins, and chain structure in their salts.

Third, the sum of the activities of the $\delta$ carbon and the active base, remains constant for the 4 salts of each of the 3 bases, but changes its value with change of base.

Fourth, by using the average value of the molecular $\alpha$ and $\gamma$ rotation constants for the three sets of salts, in connection with the sum of the molecular constants of the base and of the $\delta$ carbon, the specific rotations of the I2 salts have been computed, with an average departure from observed values of less than $0.5^{\circ}$, the average errors being as follows, viz., $0.94^{\circ}$ for the strychnine salts, $0.25^{\circ}$ for the brucine salts, and $0.22^{\circ}$ for the quinine salts, the algebraic sum of the departures for the entire series of 12 compulations being only $0.16^{\circ}$.

Fifth, the work of Dr. Hudson and his co-workers has been briefly tabulated, showing conclusively that in 30 different instances, the activity of the A carbon is not greatly changed by configuration changes in other parts of the molecule and proving that Van't Hoff's principle applies to the A carbon in many of the sugars and their derivatives.

Sixth, it has also been pointed out that this work of Dr. Hudson likewise proves that the activities of other carbons, e.g., the $\gamma$ carbon are unaffected by configuration changes on the $A$ carbon, and that Van't Hoff's principle is therefore valid for carbons other than the $A$ or terminal carbon.

Washington, D. C. 